\title{
SHARPP Games for the Education Prevention and Reversion of Chronic Diseases
}

\author{
Claris Yee Seung Chung \\ The University of Auckland \\ claris.chung@auckland.ac.nz
}

\author{
David Sundaram \\ The University of Auckland \\ d.sundaram@auckland.ac.nz
}

\begin{abstract}
Prevalence of chronic diseases has become a serious problem in our society. These illnesses are commonly caused by dietary and lifestyle risk factors and are hard to cure completely. However, behavioral changes such as adopting a healthy diet and lifestyle are effective in reducing chronic conditions. In this research, we propose to build games that could support the Sustainable transformation of chronic patients in a Holistic and Adaptable manner using Real-time, Precise, and Persuasive (SHARPP) principles, processes, systems, and technologies. This research leverages wearable information technologies and chronic disease studies to design games that interweaves virtual worlds with the real world. It not only supports patients to form healthy habits that prevent and revert chronic diseases but also helps individuals to balance various life dimensions.
\end{abstract}

\section{Background}

As populations age and chronic diseases proliferate even in the young generation, adopting a healthy lifestyle becomes a cornerstone for wellbeing. Chronic diseases are long-term, recurring health conditions that most of the world population experience in their lives. Chronic diseases have become a more serious problem as the diagnosed age is getting younger and causes $40 \%$ of premature death under the age of 70. The World Health Organization [1] estimates that chronic illnesses can cause 52 million deaths by 2030. The increase of chronic patient population also means significant economic burden and loss to both individuals and society.

Chronic conditions are commonly caused by dietary, and lifestyle risk factors, such as smoking, alcohol misuse, sedentary lifestyle and high-calorie eating habits [2], [3], and are hard to cure completely.
Dietary and lifestyle risk factors are developed gradually based on the individual's behaviors and decisions. Therefore, chronic healthcare models should be focused on prevention and reversion of diseases rather than only treating the illness and/or the symptoms. Also, it is better to shift the patient' role from a passive treatment receiver to an active self-caregiver. Due to these reasons, Chronic Diseases Self-Management Programs (CDSMP) are offered by health organizations. mHealth is an emerging industry which is viewed as antidote to transform current healthcare systems and they often address the shortcomings of physically offered selfmanagement programs [4]. Notwithstanding the proven benefits of self-management programs [5][7], the attrition rate of participations is still high [8], because of the existing problems and barriers in both patients' life and current healthcare systems [9].

To increase the efficacy of self-management programs and the sustainability of chronic patients' life, each patient's needs and circumstances would be considered holistically. Continuous support should be provided for his/her lifestyle transformation to reduce the risks of chronic conditions. In the research, we propose to build games that would support the Sustainable transformation of chronic patients in a Holistic and Adaptable manner using Real-time, Precise, and Persuasive (SHARPP) principles, systems and technologies [10]. The result would be a suite of research artifacts - models, frameworks, processes, game components and applications - that aims to fulfil our research requirements with a multimethodological design science research approach.

The remainder of this paper proceeds as follows: Section 2 discusses the motivations of our research, which encompasses the problems faced by the current CDSMP approaches and systems. In response to these issues, Section 3 proposes the core principles and processes of SHARPP that would help in addressing the identified problems. Section 4 lays out the conceptual framework of the SHARPP games, modalities and interaction between user and games, as well as the SAHRPP games architecture. Section 5 illustrates how these SHARPP games could be used 
to prevent chronic diseases by applying the principles and processes introduced in Section 3. The last Section concludes with a discussion on how the SHARPP approach could be extended to achieve the holy grail of individual sustainability and wellbeing.

\section{Motivation}

\subsection{Self-management programs and mHealth applications}

As chronic diseases are caused by lifestyle risk factors (which are modifiable), Chronic Disease SelfManagement Program (CDSMP) is often recommended to improve patient's health conditions. The main purpose of CDSMP is to teach chronic condition management skills to patients, so they can change lifestyle in living with chronic conditions. With an efficacious CDSMP, patients can increase skills and confidence in managing their health problems, including regular assessment of progress and problems, goal setting, and problem-solving support to maintain a satisfactory quality of life [11]. Many studies continuously report that unhealthy diet, physical inactivity or tobacco consumption are modifiable, and a healthy lifestyle can effectively prevent critical health conditions [12], [13]. For example, recent medical experiments and studies report that the reversal of type- 2 diabetes was examined after the appropriate medical interventions with healthy lifestyle adoption [14], [15].

Traditionally CDSMP is offered in physical places, but mobile health applications are rapidly being developed with the uptake of mobile technologies and wearable devices. According to the World Health Organization [16], "Mobile Health (mHealth) is an area of electronic health (eHealth), and it is the provision of health services and information via mobile technologies such as mobile phones and Personal Digital Assistants (PDAs)". With the help of mHealth applications, patients can easily track their bioinformatics and health-related behaviors, and communicate better with health care professionals [17]. mHealth shows clear potentials to enhance current healthcare systems and clinical interventions [4], [18]. Ubiquitous and personal mobile devices can bring convenient, cost effective healthcare to patients. Health education can be delivered to the public at a large scale, and healthcare can reach remote places easily through $\mathrm{mHealth}$.

\subsection{Issues with self-management programs and mHealth applications}

However, there are still many patients who desire to reduce risks of chronic conditions but fail to do so. The major weakness of CDSMP is a high attrition rate. A considerable proportion of participants leave the program without finishing due to various reasons [19]. The most frequently noted barriers and attrition factors are conflicting schedule, apathy toward the program, low self-efficacy, poor physician communication, low family support, financial problems and severity of symptoms like depression, difficulty in exercising, fatigue, and pains [8], [9], [20]. Despite these studies contributing to improving self-management programs in many ways; chronic patients still struggle to adhere to the programs. mHealth can improve some of these shortcomings of physically offered programs, but current mHealth applications offer limited features like text messages for notifications or multimedia for education [21]. One of the root issues of current CDSMP is that it is often prescribed to get specific outcomes like a medication for a certain period only; most of them are simply health-focused educational programs and interactions between patients and clinicians are rather episodic. Adopting new lifestyle which never take consideration of a patient's unique life requirements holistically can be a hard mission to patients. Unfortunately, current mHealth applications are rarely developed with comprehensive models and frameworks for supporting patient's life in a holistic and sustainable manner. These issues create huge challenges particularly to chronic patients, as chronic diseases are life-long conditions, unlike acute diseases. This is the reason why healthcare system should be designed and offered based on robust principles and philosophies [22].

Our research adopts the multi-methodological design science approach that has grown in prominence in the recent decades in information systems (IS) [23], [24]. This research first identifies five research requirements for an effective application in facilitating the sustainability of chronic patients. They are: (a) to support patients' sustainable lifestyle, (b) to raise patients' awareness of the multifaceted and interrelated dimensions of life, (c) to facilitate patients' adaptiveness to lifestyle changes, (d) to provide patients with tailored and timely information, and (e) to encourage patients' adherence to a sustainable lifestyle. Having identified these criteria, the research moves on to explore a variety of avenues to address them effectively, including, but not limited to, the use of models, frameworks, processes, etc. The research is guided by 
a multi-dimensional design science driven artifact oriented methodology, and aims to address the criteria in a holistic manner. In addition, to check the validity of the proposed concepts, we implement and apply the working prototypical games in the context of real world situations, devices, apps, heuristics, hacks, and dynamic social and personal contexts. Finally, evaluation of SHARPP games would be carried out based on research requirements within the framework of the "Design Evaluation Methods" suggested by Hevner et al. [24].

In the following section, we will discuss SHARPP principles and process, which are used to address the aforementioned problems and issues.

\section{SHARPP principles and processes}

\subsection{SHARPP principles}

Life is a long journey of dealing with managing and balancing many dimensions: health, wealth, careers, relationships and so on. A healthy person is formed by the integration of the spiritual, intellectual, physical, emotional, environmental and social dimensions [25]. However, balancing all these life dimensions and maintaining the quality of life can be challenging. This applies more than ever to chronic patients as their conditions cause multiple life uncertainties; chronic patients are facing a great amount of anxiety, which can harm their quality of life significantly [26]. Therefore, whether patients have skills to sustain their life while dealing with health concerns or not can be crucial. Due to this reason, healthcare promotions and educations should be developed with relevant principles.

Using principles for designing a healthcare system is particularly important. The principles help organize a various form of knowledge to design, implement and evaluate the system [27]. Mirza et al. call attention to current challenges in the global healthcare alongside with the growing ageing and chronic condition populations, and then suggest Sustainable, Holistic, Adaptive, Real-time, and Precise (SHARP) principles for developing Health and Wellness Systems [10]. Sustainability is the ability to continue and maintain one's life even if there is an imbalance of life dimensions. The sustainable individual can manage his/her life even by taking these threats as life-improving opportunities [28]. Having a holistic view of our life, which means understanding intertwined relationships among life dimensions, should be the first step to being a sustainable individual [29]. To support the sustainability vision for chronic patients, CDSMP should be adaptive, offered in real-time with precisely tailored information to each patient. SHARP principles provide solutions for the effective management of chronic patient's long-term health and wellbeing. They can also be applied various scenarios by covering a wide range of target groups.

Also, this research adds one more principle, "Persuasive" concept to develop the SHARPP games to transform the patient's lifestyle. Unfortunately, many people often become shortsighted and estrange current selves from their future selves. When their lives are filled with uncertainties, people tend to trade their future benefits with the immediate requirements [30]. This is one of the biggest attrition factors especially among younger, unmarried and lowsocioeconomic status potential patients who often risk their health to meet the current life requirements [8], [20].

\subsection{SHARPP processes}

A sustainable lifestyle can be developed through the formation of good habits and the dissolution of bad habits. Therefore, one crucial consideration in designing CDSMP applications is to understand how patients' habits are formed and dissolved. One of the well-developed behavior change theory is Transtheoretical Model of Change (TMC) [31]. This theory explains how people adapt and change gradually (i.e. pre-contemplation, contemplation, determination, action, and maintenance) until their desired behavior is attained [32]. Pre-contemplation refers to the stage that people do not realize a need of change. Contemplation refers to people who are aware of their problems but have not yet made any commitment. Preparation is the stage when people try to make small behavioral changes. In contrast to the previous stages, people in the action stage seriously adjust their behavior. Once people step into the Maintain stage, they do not need hard work to change but should reinforce actions for concrete changes. Finally, people realize that their behaviors are totally changed in the Termination stage through the measurement and monitoring of various key performance indicators.

Adopting central concepts from Transtheoretical Model of Change is particularly practical for sustainable lifestyle transformation. TMC embraces exit and relapse stages and allows entry again at any stage, the theory guides how CDMSP applications should function to help patients' lifestyle transformation. Multiple relapses and exits into old habits are part of behaviors/habit change (lifestyle transformation) processes, and the applications to support should be able to adapt and refine the 
personalization algorithms. In line with this, SHARPP games have four support stages (Sense, Interpret, Response, and Transformation) by employing the core concepts/stages of "Preparation, Action, Maintain and Termination" from TMC (Figure 1).

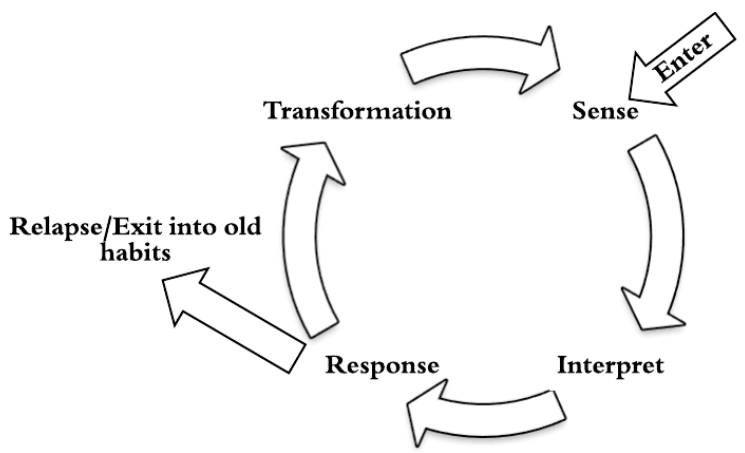

Figure 1. SHARPP processes

In the sense stage, patients get to know about current situations by measuring and collecting current multi-dimensional lifestyle data. Lifestyle data is automatically collected by wearable devices which are connected to SHARPP games. Patients' profile data, schedule information and desired habit information can be attained through direct input by users or integrated from other sources. One of the keys to successful adoption of these systems is the minimization of direct input [33]. In the interpret stage, the collected data is analyzed through multidimensional causal relationship and interdependent relationship models. These are then interpreted to design personalized lifestyle (habits) recommendations. In the response stage, patients try recommended actions to form a habit. Most of the behavioral change actions are repeatedly encouraged through persuasive techniques at this stage based on habit formation models and heuristics.

Habit is discussed as "a settled or regular tendency or practice, especially one that is hard to give up" [34]. Habits are formed through iteration of a three-step loop: cue, routine and reward [35]. Recently, neurologists also found that habits are automated responses by our brains [36]. When "cue" is presented either intrinsically or extrinsically, the brain reacts on the cue. Once the brain realizes the cue, it then starts to crave for the reward. When the habit has not formed yet, the brain is aware of the actions we are carrying out (routine). However, if the routine is repeated often, the brain converts the routine into an automated process. This is called "chunking" [36], [37]. When the brain is chunking the routine, the habit is formed. This is the reason why formed habits are hard to break. Therefore, replacing a routine is an easier way to change an old habit, rather than getting rid of it. To replace the old habit, we need to observe our behaviors to find out what are the cues, routines and rewards first, and then persuasive interventions should be continued until the old routine is replaced with the new one [35].

Once patient's behavior/habit change patterns are persistent and settled, various feedback and rewards also should be given in the transformation stage to make patient continue the changed lifestyle. Based on cumulative achievements the individual slowly undergoes transformation. In the next section, a conceptual framework, modalities and interactions, and the architecture of SHARPP games are discussed to elucidate the behavioral aspects of users and games.

\section{SHARPP games}

\subsection{SHARPP games conceptual framework}

For preventing and reversing chronic diseases, we suggest "SHARPP games" which could help patients to manage their diseases and live better and easier. The game is a well-studied educational tool and good at engaging audiences with interesting graphical presentation and multimedia [38]. Game-based learning is more effective and motivates students better than traditional learning [39]. Therefore, online games are often used for teaching young students how to prevent chronic diseases. Utilizing games to address chronic diseases can reduce patient's resistance to change by increasing perceived benefits and values. Samhan [40] conducted a study on why people are resistant to using the Patient Portal System. One of the key findings indicates that patients are willing to use the portal system, and minimize their resistance to change, if they see more benefits to using the system. This industry is still in its infancy, but the number of experimental online game projects in chronic disease education is increasing. DeShazo, Harris and Pratt [41] conducted a literature review for 11 diabetes education video games from nine studies, and concluded that online games could be effective educational interventions. Currently, most health online games are targeting children [41], in fact, the average age of gamers is 35 years old [42], and $26 \%$ of gamers are 50 years and older in United States [43]. This fact infers that there is a niche opportunity for elderly gamers.

SHARPP games are designed to deliver the objectives for transforming chronic patient's lifestyle. The purposes of the games are primarily for 
education, secondly for prevention, and thirdly for reversion of chronic diseases where possible. Education often results in prevention of chronic disease, and as education helps us to prevent, we get more to know about ourselves better what works for us and what does not. And the same thing applies where games are helping us to rehabilitate current chronic conditions, we also learn ourselves better, and the cycle goes on (Figure 2).

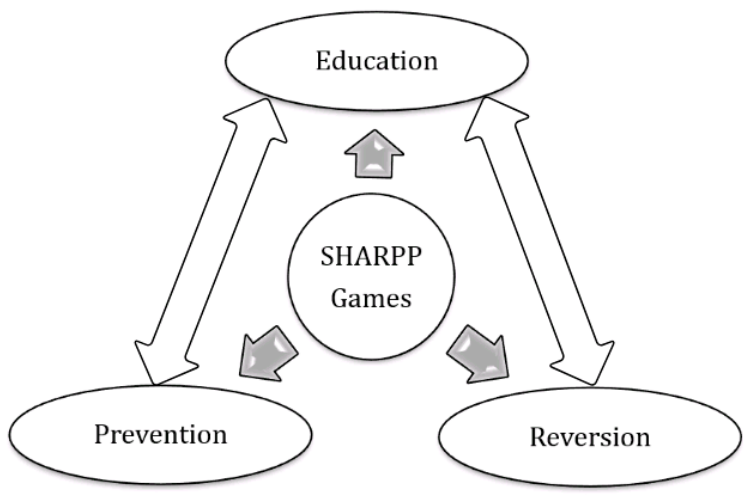

Figure 2. SHARPP games framework

The games are primarily driven through recommender systems based engine. They will recommend different action choices and possibilities depending on their purpose: education, prevention or reversion. Also, the games will personalize recommendations based on each patient's unique needs and circumstances, such as age, family history, job, and life patterns so on. For example, if a patient already practices serious exercise but yet to improve chronic condition, then the games would recommend a diet to change after analyzing collected data and information.

\subsection{SHARPP games modalities and interactions}

The games have various modalities and interaction mechanisms - from the purely virtual to the completely real and a whole plethora of combinations in between as illustrated in Figure 3. In the pure game layer, the games are composed of various worlds representing our life dimensions. For example, health world teaches and helps patients to adopt the healthy diet, exercise, rest and stress managing habits and lifestyle. Each world uses relevant themes and scenarios to help patients manage their illnesses and lives better and easier. Also, these worlds can be integrated together to deliver the advanced educational health interventions.
In the real-world layer, the games use persuasive gamification features to encourage patients act on the sustainable lifestyle. In this layer, the games are working as measurement, management and benchmarking systems for changing the patient's behaviors and attitudes towards healthy lifestyle through persuasive technologies [44]. The games also bridge the pure game and the real-world layers.

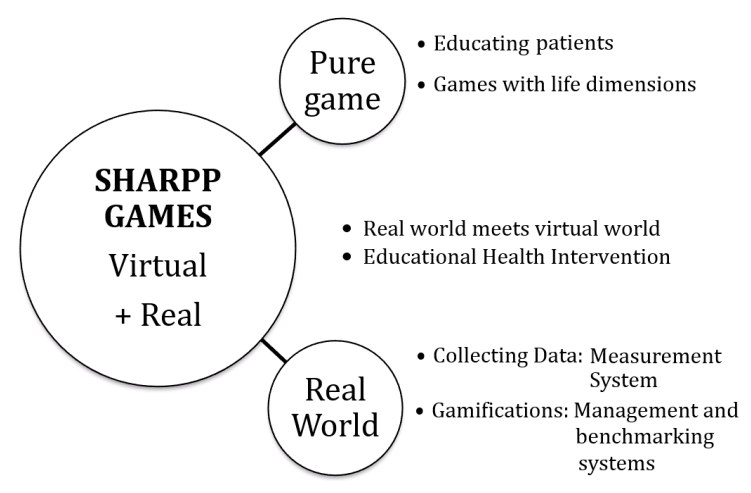

Figure 3. SHARPP games modalities and interactions - from virtual to the real

The SHARPP games user interaction happens in real-time. Once a user initiates the SHARPP games for the first time, the games will ask the user to input relevant data, such as demographic, and health data in the "Profile Setup" page. After this, the user can choose the purpose of using the games: education, prevention or reversion. Then the games will show "Wearable and Smart Devices Connection" page to connect external devices which will help the user to $\log /$ collect their data automatically. According to an empirical investigation on diabetes self-management mobile application reviews, automatic logging/data collection is one of the essential features to lower the users anxiety and frustration of record keeping [33]. Therefore, the integration between wearable devices and SHARPP games is vital. Depending on the purpose of the games, different devices will be shown in the connection page. This is because particular devices for certain diseases (e.g. glucose monitors or cardiac monitoring devices) are not needed to be connected/integrated for users who would like to use the games for purely educational purposes. As illustrated in Figure 4, the games user interaction calibrates games based on needs and circumstances. Once the personalized game is chosen and ready, the user can play games in a way that interweaves both the real world and the virtual world. For instance, if the user plays a walking game to form a habit, then as the user walks in real-life, the game shows a character walking in the game too. Or based on the 
user's actual walking steps (collected automatically via wearable devices), the game character shows its action in a fast-forward manner when the user initiates the game. During the day, the SHARPP games keep monitoring the user's actions and sending persuasive notifications to motivate the user. For instance, game items are given as rewards if the user plays the game regularly. The game will daily update user's data and report the result back to the user. After playing games, the user can continue to play the same game to form a desired habit or choose other games if it does not fit his/her needs and circumstances. Through these iterations, the user is getting to know about his/her lifestyle/habits better, while being educated and entertained by the SHARPP games. In other words, SHARPP games help the user to find and transform lifestyle through game play.

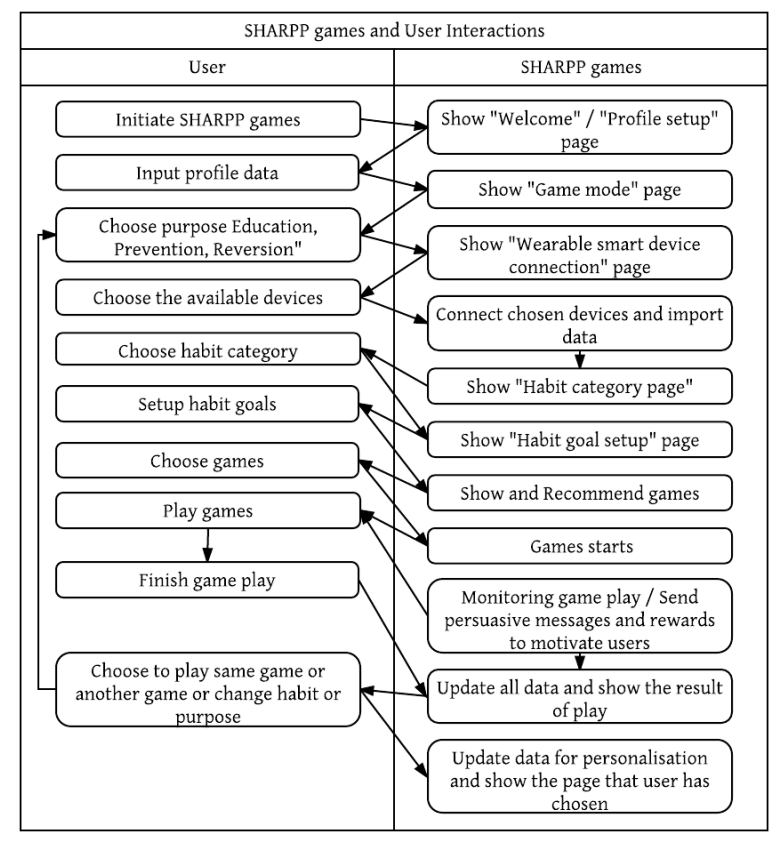

Figure 4. SHARPP games user interactions

\subsection{SHARPP games architecture}

The SHARPP games architecture outlines the structure and functions of games and provides guidance for implementation (Figure 5). Through the interface layer, users can (a) configure a vision, goals, profile, health, habit, schedule, and games; (b) manage health, habit, and schedule transactions and (c) play games. The transactions are automatically updated via wearable devices, social media and game plays as much as possible. To support the sustainable lifestyle transformation, data (clinical data, personal data, and environment \& social data), models, algorithms, methods, solvers, visualization, scenarios, rules, heuristics and knowledge are located in the component layer of the architecture. The middle level is the application layer which offers game, knowledge, lifestyle, and recommendation management. Components in the component layer and management services in the application layer communicate and cooperate together to provide precise personalized games. For example, if the user wants to form a healthy diet habit with a low budget constraint, lifestyle and knowledge management modules will retrieve relevant data to analyze them through multi-dimensional causal relationship and interdependent relationship models. Then game management design will personalize the habit formation games based on recommendations from the recommendation management module.

Wearable devices and Social media are connected to both users and SHARPP games to collect data and information. Especially, the direct connection/integration of social media to users provides social and peer support which can be the most effective persuasive technique [33] in SHARPP games.

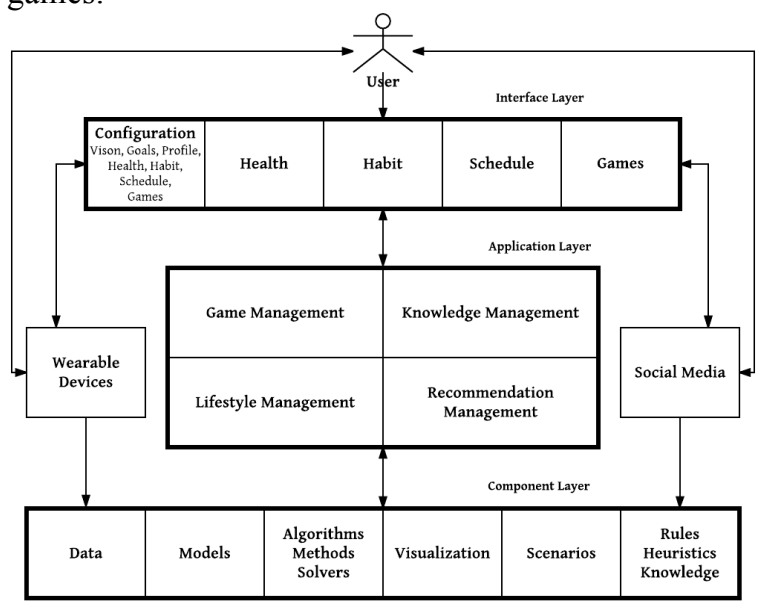

Figure 5. SHARPP games architecture

\section{SHARPP games for the education prevention and reversion of chronic diseases}

\subsection{Sustainable}

Pappas [28] suggests that "Sustainable individuals are characterized by creating harmony, interconnection, and relatively high levels of selfawareness in their values, thoughts, behaviors, and actions, as well as cultivating continued individual growth in their physical (health), emotional, social, 
philosophical, and intellectual abilities." In other words, the concept of individual sustainability is one that enables individuals and families to be well, or happy while they are balancing various life dimensions and challenges. Human's life is composed of various life dimensions, such as health, career, education, family relationship, and social status (Figure 6).

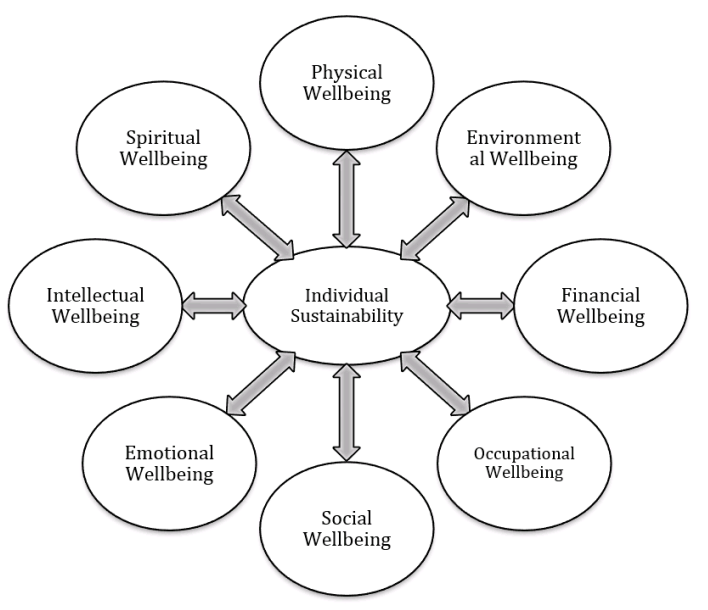

Figure 6. Multiple life dimensions for individual sustainability

Life dimensions are interrelated highly each other. Therefore, the boundary between each life dimensions is interdependent. Broadly speaking, life dimensions can be categorized based on the roles which people play in daily life [45], and they are essential elements of individuals and families to live an ordinary sound life. In addition to this interdependency, each life dimension influences each other and hence often establishes a causal relationship. For instance, it is a well-known fact that the obesity rate is higher in low socioeconomic families and it is proven by many clinical experiments from various countries [46]-[51]. Some studies suggest that adopting healthy behaviors and diet lower the medical expenditures and increase the daily productivities [47], [52], [53]. Due to these reasons, it is important to 1 ) identify different life dimensions, 2) understand and develop a holistic view of causal and interdependent dynamic intertwined models between life dimensions.

\subsection{Holistic}

Chronic patients' key concern is their health conditions, but there are many other life dimensions that they need to take care of. Lost productivity and costs of healthcare easily hurt patients and their families' financial status. Hence, they are more vulnerable to impoverishment [26]. This stress can impact on their social and family relationships and make their health condition worse.

Holistic information in an appropriate visualization can lower the chronic patients' anxiety level and strengthen the engagement on treatments [54], [55]. As such, once SHARPP games collect the chosen lifestyle dimension data by patient's virtual and real-world actions, the relationship of the patient's life dimensions is presented in "Radar diagram" format in the games. The radar diagram can show multiple lifestyle dimensions in one picture and explains the relationship among dimensions (Figure 7). This visualization happens after the games use multiple models to analyze heterogeneous data from various sources. Models for recommendation and behavioral changes can be implemented using solvers, such as mathematical and recommendation algorithms, data mining techniques and system dynamics.

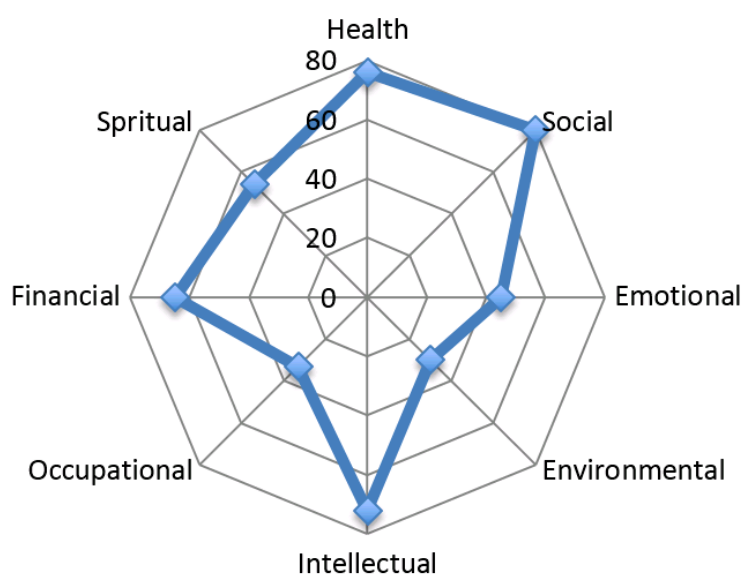

Figure 7. Holistic visualization

\subsection{Adaptive}

Broadly speaking, transforming chronic patient's life to be sustainable is a whole process of adaptation. Chronic patients are continuously trying different lifestyle patterns to find the one works for them. Therefore, the entire SHARPP games are designed based on the "adaptive" concept in mind. For example, SHARPP processes allows "exit and enter" at any stage for multiple tryouts by patients. Through these tryouts, the patients get to know more about themselves and the games also learn what works and what does not by collecting and analyzing patients' tryout data. Another example of "adaptive" concept in the games is habit formation. As mentioned before, the habit has 3-step process: cue, routine, and reward. 
Based on this process, the games teach patients how to utilize existing habit's reward as a cue for another habit. In other words, teaching patients to chain positive habits up together. Let's say there is a person who showers (routine) before going to bed (time: cue) because he/she likes fresh feeling in the bed (reward). The games can teach to add one more habit into this existing one: taking "fresh feeling in the bed" as a new cue for 5-minutes meditation on the bed (a new routine), then "feeling fresh and relaxed for better sleep" can be the new reward. When patients can chain up positive habits in the real world, the games award bonus score or virtual gifts to patients and visualize how these habits can benefit the future patient's life.

\subsection{Real-time, precise, and persuasive}

Health behavior changes do not take place in a vacuum. To provide precise information to patients, at least three levels of data should be collected: clinical data, profile data, and environment \& social data. Clinical data is produced from the clinical environments such as lab results, medical research, and information about diseases. Personal data includes personal health data, lifestyle patterns, family situations and so on. The environment \& social data includes environmental and social data such as climate, community safety, food or exercise availability and so on. These types of data will be collected in real-time through electronic health records, wearable devices, smart health monitoring devices and publically available social information, etc. By processing and analyzing them, the games can predict the potential health threats and alert patients to take actions for it. While patients are acting on the recommended actions, the games use persuasive technologies to encourage patients in realtime so that they can adhere to the actions for behavior changes.

\section{Individual sustainability and wellbeing: The Holy Grail}

Chronic illnesses are caused by lifestyle risk factors, and the research has proven the selfmanagement programs are effective interventions to reduce chronic condition mortality and enhance the patients' health outcomes. Current mobile technology development makes the self-management industry to provide ubiquitous interventions at the lower cost. However, the absence of a holistic model that embraces relationships between such risk factors and chronic diseases' cues resulted in mobile health applications suffering from a high attrition rate.

SHARPP games are specially designed to educate chronic patients. They are the mobile educational intervention games that provide comprehensive information about chronic conditions and sustainability based on a holistic approach and assists patients' lives better and easier. Also, striking motivation features are employed to support patients' actions. SHAPP approach was originally derived from SHARP principles which was suggested for Health and Wellness Systems to manage individuals' long-term health and well-being [10]. Therefore, SHARPP games can be utilized not only for chronic patients but also individuals who want to transform their life using fun as a motivator. The purpose of the games is helping individual's life become more sustainable through holistic understanding. All game scenes provide useful information and skills to help individual's life with current conditions. Based on these, educate individuals to choose healthy options in each situation and encourage them to take healthy options in their real lives. Also, the games will suggest personalized options based on the collected personal data and requirements for each individual.

Currently, most of the game-based educational intervention projects are targeting children and the younger generation, but game-based educational interventions would be effective in the adult population as the average online gamers age is 35 years. Therefore, we believe that SHARPP Game can enlighten not only chronic condition patients but also individuals to achieve the holy grail of sustainability and wellbeing.

\section{References}

[1] C. Wing and H. Yang, "FitYou: Integrating Health Profiles to Real-Time Contextual Suggestion," in Proceedings of the 37 th international ACM SIGIR conference on Research \& development in information retrieval - SIGIR '14, 2014, pp. 1263-1264.

[2] J. M. McGinnis and W. H. Foege, "Actual Causes of Death in the United States," J. Am. Med. Assoc., vol. 270, no. 18, pp. 2207-2212, 1993.

[3] A. H. Mokdad, J. S. Marks, D. F. Stroup, and J. L. Gerberding, "Actual causes of death in the United States, 2000," J. Am. Med. Assoc., vol. 291, pp. 1238-1245, 2004.

[4] W. a Kaplan, "Can the ubiquitous power of mobile phones be used to improve health outcomes in developing countries?," Global. Health, vol. 2, p. 9, 2006.

[5] S. Newman, L. Steed, and K. Mulligan, "Self- 
management interventions for chronic illness," Lancet, vol. 364, no. 9444, pp. 1523-1537, 2004.

[6] K. R. Lorig, D. S. Sobel, A. L. Stewart, B. W. Brown, A. Bandura, P. Ritter, V. M. Gonzalez, D. D. Laurent, and H. R. Holman, "Evidence Suggesting That a Chronic Disease Self-Management Program Can Improve Health Status While Reducing Hospitalization," Med. Care, vol. 37, no. 1, pp. 5-14, 1999.

[7] K. R. Lorig, D. S. Sobel, P. L. Ritter, D. Laurent, and M. Hobbs, "Effect of a Self-Management Program on Patients with Chronic Disease," Eff. Clin. Pract., vol. 4, no. 6, 2001.

[8] E. Gucciardi, M. Demelo, A. Offenheim, S. L. Grace, and D. E. Stewart, "Patient factors associated with attrition from a self-management education programme," J. Eval. Clin. Pract., vol. 13, no. 6, pp. 913-919, 2007.

[9] A. F. Jerant, M. M. Von Friederichs-Fitzwater, and M. Moore, "Patients' perceived barriers to active selfmanagement of chronic conditions," Patient Educ. Couns., vol. 57, no. 3, pp. 300-307, 2005.

[10] F. Mirza, A. Mirza, C. Y. S. Chung, and D. Sundaram, "Sustainable, Holistic, Adaptable, Real-Time, and Precise (SHARP) Approach Towards Developing Health and Wellness Systems," nternational Conf. Futur. Netw. Syst. Secur., vol. 670, pp. 157-171, 2016.

[11] J. Trappenburg, N. Jonkman, T. Jaarsma, H. van OsMedendorp, H. Kort, N. de Wit, A. Hoes, and M. Schuurmans, "Self-management: One size does not fit all," Patient Educ. Couns., vol. 92, no. 1, pp. 134-137, 2013.

[12] Centers for Disease Control and Prevention, "Sorting through the Evidence for the Arthritis Self-Management Program and the Chronic Disease Self-Management Program. Executive Summary of ASMP/CDSMP MetaAnalyses," 2011.

[13] E. Johnston, S., Liddy, C., Ives S. M., Soto, "Literature Review on Chronic Disease Self-Management," pp. 1-26, 2008.

[14] D. M. Dunaief, J. Fuhrman, J. L. Dunaief, and G. Ying, "Glycemic and cardiovascular parameters improved in type 2 diabetes with the high nutrient density (HND) diet," Open J. Prev. Med., vol. 2, no. 3, pp. 364-371, 2012.

[15] E. L. Lim, K. G. Hollingsworth, B. S. Aribisala, M. J. Chen, J. C. Mathers, and R. Taylor, "Reversal of type 2 diabetes: Normalisation of beta cell function in association with decreased pancreas and liver triacylglycerol," Diabetologia, vol. 54, no. 10, pp. 2506-2514, 2011.

[16] World Health Organization, "World Health Statistics, $2011, " 2011$.

[17] K. Patrick, W. G. Griswold, F. Raab, and S. S. Intille, "Health and the Mobile Phone," Am. J. Prev. Med., vol. 35, no. 2, pp. 177-181, 2009.

[18] M. N. K. Boulos, S. Wheeler, C. Tavares, and R. Jones, "How smartphones are changing the face of mobile and participatory healthcare: an overview, with example from eCAALYX.," Biomed. Eng. Online, vol. 10, no. 1, 2011.

[19] N. Verevkina, Y. Shi, V. A. Fuentes-Caceres, and D. P. Scanlon, "Attrition in Chronic Disease Self-Management Programs and Self-Efficacy at Enrollment," Heal. Educ. Behav., vol. 41, no. 6, pp. 590-598, 2014.

[20] E. Gucciardi, M. Demelo, A. Offenheim, and D. E. Stewart, "Factors contributing to attrition behavior in diabetes self-management programs: a mixed method approach.," BMC Health Serv. Res., vol. 8, p. 33, 2008.

[21] C. Free, G. Phillips, L. Galli, L. Watson, L. Felix, P. Edwards, V. Patel, and A. Haines, "The Effectiveness of Mobile-Health Technology-Based Health Behaviour Change or Disease Management Interventions for Health Care Consumers: A Systematic Review," PLoS Med., vol. 10, no. $1,2013$.

[22] R. R. Cottrell, J. T. Girvan, J. F. McKenzie, and D. Seabert, Principles and foundations of health promotion and education, 7th Revise. New Jersey, United States: Prentice Hall, 2017.

[23] J. Nunamaker, M. Chen, and T. Purdin, "Systems Development in Information Systems Research," Journal of Management Information Systems, vol. 7., 1991.

[24] A. R. Hevner, S. T. March, J. Park, and S. Ram, "Design Science in Information Systems Research," MIS $Q$., vol. 28, no. 1, pp. 75-105, 2004.

[25] R. J. Donatelle and L. G. Davis, Health: The basics, Green Edit. San Francisco: Pearson Education, 2011.

[26] H. Kankeu, P. Saksena, K. Xu, and D. B. Evans, "The financial burden from non-communicable diseases in lowand middle-income countries: a literature review," Heal. Res. Policy Syst., vol. 11, no. 1, p. 31, 2013.

[27] R. A. Crosby, L. F. Salazar, and R. J. DiClemente, "How theory informs health promotions and public health practice," in Health behavior theory for public health: Principles, foundations, and application, Burlington, MA: Jones \& Bartlett Learning, 2013, pp. 22-44.

[28] E. Pappas, O. Pierrakos, and R. Nagel, "Using Bloom's Taxonomy to teach sustainability in multiple contexts," J. Clean. Prod., vol. 48, pp. 54-64, 2013.

[29] C. Y. S. Chung, R. Proskuryakov, and D. Sundaram, "Design and Implementation of Sustainable Social Shopping Systems," in Nature of Computation and Communication, vol. 144, 2015.

[30] H. Ersner-Hershfield, M. T. Garton, K. Ballard, G. R. 
Samanez-Larkin, and B. Knutson, “Don't stop thinking about tomorrow: Individual differences in future selfcontinuity account for saving.," Judgm. Decis. Mak., vol. 4, no. 4, pp. 280-286, 2009.

[31] J. O. Prochaska, C. C. DiClemente, and J. C. Norcross, "In search of how people change. Applications to addictive behaviors.," Am. Psychol., vol. 47, no. 9, 1992.

[32] J. A. Hayden, Introduction to health behavior theory, 2nd Editio. Burlington, MA: Jones \& Bartlett Learning, 2013.

[33] M. Dadgar and K. D. Joshi, "Diabetes SelfManagement Using Mobile Apps: An Empirical Investigation Based On App Reviews And Through Value Sensitive Design Perspective," in 2015 International Conference on Mobile Business, 2015.

[34] OxfordDictionaries, Oxford Dictionary of English, 7th Revise. Oxford, United Kingdom: Oxford University Press, 2013.

[35] C. Duhigg, The Power of Habit: Why we do what we do and how to change. New York: Random House, 2012.

[36] H. H. Yin and B. Knowlton, "The role of the basal ganglia in habit formation.," Nat. Rev. Neurosci., vol. 7, no. 6, pp. 464-76, 2006.

[37] A. M. Graybiel, "Building action repertoires: Memory and learning functions of the basal ganglia," Current Opinion in Neurobiology, vol. 5, no. 6. pp. 733-741, 1995.

[38] S. de Freitas and M. Griffiths, "Online gaming as an educational tool in learning and training," $B r . J$. Educ. Technol., vol. 38, no. 3, pp. 535-537, 2007.

[39] M. J. Dondlinger, "educational video game design: a review of the literature," J. Appl. Educ. Technol., vol. 4, no. 1, pp. 21-31, 2007.

[40] B. Samhan, "Why Do People Resist Patient Portal Systems?: An Application of the Dual Factor Model of IT Usage," Int. J. Healthc. Inf. Syst. Informatics, vol. 12, no. 4, pp. 68-86, 2017.

[41] J. Deshazo, L. Harris, and W. Pratt, "Effective Intervention or Child's Play? A review of video games for diabetes education," Diabetes Technol. Ther., vol. 12, no. 10, pp. 815-822, 2010.

[42] W. Ijsselsteijn, H. H. Nap, Y. de Kort, and K. Poels, "Digital Game Design for Elderly Users," Proc. 2007 Conf. Futur. Play, pp. 17-22, 2007.

[43] Statista, "Age breakdown of video game players in the United States in 2017," 2017. [Online]. Available: https:/www.statista.com/statistics/189582/age-of-us-videogame-players-since-2010/. [Accessed: 01-May-2017].
[44] B. J. Fogg, "Persuasive Computers: Perspectives and Research Directions," in Proceedings of the SIGCHI conference on Human factors in computing systems, 1998.

[45] D. T. Hall and J. Richter, "Balancing Work Life and Home Life: What Can Organizations Do to Help?," Acad. Manag. Exec., vol. 2, pp. 213-223, 1988.

[46] R. Devol and A. Bedroussian, "An Unhealthy America: The Economic Burden of Chronic Disease," Stroke, no. October, 2007.

[47] E. a. Finkelstein, I. C. Fiebelkorn, and G. Wang, "National medical spending attributable to overweight and obesity: how much, and who's paying?," Health Aff. (Millwood)., vol. Suppl, 2003.

[48] L. Nasreddine, F. Naja, C. Akl, M. C. Chamieh, S. Karam, A. M. Sibai, and N. Hwalla, "Dietary, lifestyle and socio-economic correlates of overweight, obesity and central adiposity in lebanese children and adolescents," Nutrients, vol. 6, no. 3, pp. 1038-1062, 2014.

[49] T. Pouliou and S. J. Elliott, "Individual and socioenvironmental determinants of overweight and obesity in Urban Canada," Heal. Place, vol. 16, no. 2, 2010.

[50] M. Wake, P. Hardy, L. Canterford, M. Sawyer, and J. B. Carlin, "Overweight, obesity and girth of Australian preschoolers: prevalence and socio-economic correlates," Int. J. Obes., vol. 31, no. 7, pp. 1044-1051, 2007.

[51] B. Xie, C. P. Chou, D. Spruijt-Metz, K. Reynolds, F. Clark, P. H. Palmer, P. Gallaher, P. Sun, Q. Guo, and C. A. Johnson, "Socio-demographic and economic correlates of overweight status in Chinese adolescents," Am. J. Health Behav., vol. 31, no. 4, pp. 339-352, 2007.

[52] S. Arora, "Health, Human Productivity, and LongTerm Economic Growth,” J. Econ. Hist., vol.61, no.3, 2001.

[53] J. E. Riedel, W. Lynch, C. Baase, P. Hymel, and K. W. Peterson, "The effect of disease prevention and health promotion on workplace productivity: A literature review.," American Journal of Health Promotion, vol. 15, no.3, 2001.

[54] A. Coulter and J. Ellins, "Effectiveness of strategies for informing, educating, and involving patients," $B M J$, vol. 335, no. 7609, 2007.

[55] L. Y. Kiyohara, L. K. Kayano, L. M. Oliveira, M. U. Yamamoto, M. M. Inagaki, N. Y. Ogawa, P. E. S. M. Gonzales, R. Mandelbaum, S. T. Okubo, T. Watanuki, and J. E. Vieira, "Surgery information reduces anxiety in the pre-operative period.," Rev. Hosp. Clin. Fac. Med. Sao. Paulo., vol. 59, no. 2, pp. 51-56, 2004. 